\title{
Transatlantica
}

Revue d'études américaines. American Studies Journal

\section{De la porte de Brandebourg à La Havane : Wilder, Hitchcock et l'héritage révolutionnaire}

Julie Michot

\section{(2) OpenEdition}

\section{Journals}

Édition électronique

URL : https://journals.openedition.org/transatlantica/11864

DOI : $10.4000 /$ transatlantica. 11864

ISSN : 1765-2766

Éditeur

Association française d'Etudes Américaines (AFEA)

Référence électronique

Julie Michot, « De la porte de Brandebourg à La Havane: Wilder, Hitchcock et l'héritage

révolutionnaire », Transatlantica [En ligne], 1 | 2018, mis en ligne le 01 septembre 2019, consulté le 04

février 2023. URL : http://journals.openedition.org/transatlantica/11864 ; DOI : https://doi.org/

10.4000/transatlantica. 11864

Ce document a été généré automatiquement le 4 février 2023.

\section{(c) (i) $\odot$}

Creative Commons - Attribution - Pas d'Utilisation Commerciale - Pas de Modification 4.0 International - CC BY-NC-ND 4.0

https://creativecommons.org/licenses/by-nc-nd/4.0/ 


\title{
De la porte de Brandebourg à La Havane : Wilder, Hitchcock et l'héritage révolutionnaire
}

\author{
Julie Michot
}

1 Billy Wilder et Alfred Hitchcock sont deux réalisateurs qui n'ont pas la réputation de produire des œuvres à forte teneur politique, et qui n'ont jamais revendiqué ouvertement leur soutien à un quelconque parti. Wilder, en effet, est surtout célèbre pour ses prises de position sociales ou éthiques: il fustige par exemple la bêtise humaine dans Ace in the Hole (Le Gouffre aux chimères, 1951), et vilipende l'usine à rêves hollywoodienne dès le début de sa carrière avec Sunset Boulevard (Boulevard du Crépuscule, 1950), pour récidiver bien plus tard dans Fedora (1978). Certes, depuis le décès d'Hitchcock, des spécialistes se sont intéressés à la dimension idéologique de ses films. Dans son étude du réalisateur en tant que cinéaste politique, Ina Rae Hark (1990) évoque notamment Foreign Correspondent (Correspondant 17, 1940), Saboteur (Cinquième Colonne, 1942) ou encore Lifeboat (1943), c'est-à-dire des longs métrages sortis pendant le second conflit mondial, habituellement considérés comme des objets de propagande anti-nazie et comme la contribution de leur réalisateur à l'effort de guerre; en revanche, bien qu'Ina Rae Hark fasse allusion à des productions postérieures d'Hitchcock, elle ne se penche pas sur Topaz (L'Étau, 1969), un film pourtant d'autant plus intéressant qu'il est habituellement décrié, à l'instar de One, Two, Three (Un, deux, trois, 1961) de Wilder.

One, Two, Three et Topaz ont été tournés en pleine guerre froide et ont en commun de mettre en scène des capitalistes et des communistes à Berlin-Est et à Berlin-Ouest d'une part, en Europe occidentale, à New York et à Cuba d'autre part. Malgré un ton résolument burlesque et des personnages parfois caricaturaux à l'extrême qui placent son scénario à la limite de la comédie screwball, One, Two, Three n'en reste pas moins une critique sans concessions de la révolution soviétique et des régimes qui en ont découlé. Topaz d'Hitchcock, en revanche, est avant tout un récit d'espionnage au ton bien plus sérieux ; adapté d'un roman inspiré de faits réels, son action se déroule en 1962, et le 
film se clôt d'ailleurs sur la fin de la crise des missiles de Cuba, donc sur le souvenir d'une victoire - relative - des États-Unis sur l'URSS. Le film d'Hitchcock, pourtant, fait lui aussi usage de la caricature: en jouant sur les stéréotypes pour présenter l'organisation des sociétés russe et cubaine, et en grossissant le trait s'agissant des personnages de communistes, le script conforte le public américain d'origine dans ses certitudes et lui donne à voir ce qu'il a envie de voir - du moins est-ce ce qu'il ressort d'un premier visionnage.

3 Pourtant, une seconde lecture des deux œuvres révèle une dimension plus inattendue : les dialogues, omniprésents dans One, Two, Three, chargent autant les partisans de Khrouchtchev que les amoureux du tout-puissant dollar et de la société de consommation, et le dénouement fait du héros américain l'arroseur arrosé, battu sur son propre terrain par un jeune communiste. Quant à Hitchcock, il s'offre le luxe, grâce à des séquences d'archives, de faire figurer au casting plusieurs stars de la révolution cubaine dont Che Guevara, mort prématurément deux ans avant la sortie de Topaz. Le charisme du guérillero, déjà un mythe, rendrait presque la réalité (révolutionnaire) plus séduisante que la fiction (hitchcockienne), les acteurs choisis pour interpréter les agents occidentaux manquant majoritairement de relief. Si l'on ajoute à cela que, dans Topaz, la diégèse met à mal deux valeurs américaines par excellence, la famille et la patrie, et que l'épilogue du film d'Hitchcock, comme celui du film de Wilder, suggère un certain retour au point de départ, on commence à mesurer la part de subversion contenue dans les deux œuvres.

4 Les deux réalisateurs ne cèdent pourtant pas à la facilité et se refusent à donner une vision clivée des blocs impliqués dans la guerre froide : en aucun cas leur mise en scène ne présente le capitalisme comme un système sans faille, et le communisme comme une doctrine entièrement néfaste. Leurs prises de position, bien plus complexes et nuancées, passent non seulement par le cadrage, la musique, le jeu des acteurs ou les costumes, mais aussi par les liens évidents que les spectateurs ne manquent pas d'établir à la fois avec des longs métrages de fiction antérieurs, et avec des événements historiques récents. Avec One, Two, Three et Topaz, Wilder et Hitchcock, qui semblent vouloir avant tout divertir leur public avec une comédie ou un suspense, portent en fait un regard ironique sur la révolution et le monde qui les entoure en proposant deux scénarios en forme de pirouette.

\section{Un contexte de tournage tendu pour Hitchcock comme pour Wilder}

5 À première vue, ces deux films tournés à Hollywood par des réalisateurs d'origine européenne n'ont vraiment rien de révolutionnaire, que ce soit par leur scénario ou leur esthétique. One, Two, Three de Wilder est en noir et blanc alors qu'il sort en 1961, à une époque où, depuis plusieurs années déjà, le cinéma se raccroche habituellement au procédé Technicolor pour concurrencer la télévision; un tel choix de la part de Wilder peut être considéré comme une sorte de retour en arrière, voire comme un refus délibéré de combler les attentes des spectateurs, ce qui constitue une certaine prise de risque. Certes, Topaz, produit huit ans plus tard, est bien en couleurs, mais Patrick Humphries rappelle qu'Hitchcock, à 70 ans, est un homme fatigué au moment du tournage de ce film, l'un des derniers de sa carrière (le 51 $1^{\mathrm{e}}$ sur 53 ); il n'a plus le même succès auprès du public et des critiques, sans doute parce que la société change de 
manière radicale : 1969, année de la sortie de Topaz, est également celle de Woodstock, et voit paraître sur les écrans des films comme Easy Rider (Dennis Hopper), Midnight Cowboy (Macadam Cowboy, John Schlesinger) ou Alice's Restaurant (Arthur Penn). Les jeunes cinéastes du Nouvel Hollywood sont en passe de prendre le pouvoir en bouleversant les codes. Mais Hitchcock, qui a fait ses débuts en Angleterre dans les années 20, reste fidèle à lui-même au risque de paraître démodé (Humphries, 1987, 165-166). Bien qu'il s'en soit rendu compte, il semble impuissant à régler cette difficulté : après avoir visionné Blow-Up (Michelangelo Antonioni, 1966), il déclare: "Ces metteurs en scène ont un siècle d'avance sur moi au niveau de la technique. Je me demande ce que j'ai fait de tout ce temps » (Bourdon, 2007, 313).

En dépit de ses doutes, Hitchcock se lance dans la réalisation de Topaz alors même que son précédent film, Torn Curtain (Le Rideau déchiré, 1966), qui traite également d'espionnage et des relations Est-Ouest, a connu un tournage compliqué et reçu un accueil particulièrement froid. Le conflit vietnamien s'enlise, la contestation enfle aux États-Unis, et Hitchcock choisit malgré tout d'adapter un roman de Leon Uris ${ }^{1}$ datant de 1967 mais qui s'intéresse à une crise révolue, celle des missiles, remontant à 1962. Étrangement, les problèmes ne sont pas liés à l'impossibilité de tourner à Cuba mais à la position du ministre de la Culture André Malraux qui refuse à Hitchcock l'autorisation de filmer sur le sol français. Le roman de Uris a en effet été interdit en France car il relate "la présence d'un agent communiste dans l'entourage du général de Gaulle » (Truffaut, 1997, 281). Il faudra toute la diplomatie de l'ambassadeur américain à Paris pour que Malraux revienne sur sa décision (Bourdon, 2007, 314).

7 La politique poursuit également Wilder, mais de manière plus inattendue. Wilder tourne One, Two, Three à l'été 1961 à Berlin, ville occupée mais dans laquelle on passe encore assez facilement la porte de Brandebourg, dans un sens comme dans l'autre. Toutefois, le scénario est un problème pour les autorités de Berlin-Est, comme celui d'Hitchcock l'est pour le gouvernement français. Wilder, qui refuse catégoriquement de communiquer son script, doit donc ruser pour filmer aux abords du monument côté Alliés - notamment la scène dans laquelle un personnage s'apprête à entrer dans la zone soviétique avec un slogan anti-russe bien visible à l'arrière de son side-car. Aucune scène ne peut être tournée à Berlin-Est, mais cela n'a pas grande importance : les quartiers non reconstruits du secteur ouest font parfaitement l'affaire (Wood, 2001, 36-37).

8 C'est cependant un contretemps bien réel qui surgit à la mi-août lorsque, sans préavis, le Mur de Berlin est érigé en l'espace d'une nuit, comme une mauvaise blague que la RDA ferait à Wilder. Cette fois, la réalité politique dépasse la fiction et toute l'équipe du tournage est contrainte de quitter la ville pour s'installer à Munich ; on y construit une réplique de la porte de Brandebourg côté Est, ce qui fait exploser le budget du film sans que ces dépenses ne soient amorties par la suite. En effet, One, Two, Three est une pure comédie censée se dérouler quelques mois à peine avant la construction du Mur; or, dès l'apparition de celui-ci, des drames se jouent quotidiennement à Berlin où des personnes sont abattues en tentant de passer à l'Ouest. De ce fait, lorsque le film sort en décembre 1961, les spectateurs, aux États-Unis ou ailleurs, n'ont pas envie de rire de la situation (Phillips, 2001, 257-258), et le long métrage est un échec.

9 I.A.L. Diamond, co-scénariste de Wilder, explique que le monde avait changé si vite qu'ils n'avaient pas pu complètement adapter le scénario à cette nouvelle donne. Au moment où l'écriture du script a débuté, il était courant de faire des plaisanteries à 
propos de Khrouchtchev ou de Castro ; au début de l'année 1961, avec des événements comme la mort de Lumumba ou le débarquement de la baie des Cochons, le climat politique est devenu tendu et certains sujets ont soudain cessé d'être drôles (Madsen, 1969, 46). Wilder utilise une métaphore très parlante pour évoquer ces difficultés: «C'était comme faire un film à Pompéi avec toutes les coulées de lave. Khrouchtchev allait encore plus vite que Diamond et moi. Nous devions constamment modifier le scénario pour rester raccord avec l'actualité »(Lally, 1996, 317, je traduis)². Wilder déclare également :

Les réalisateurs sont vulnérables à ce type de risque. Il suffit que la situation ou le climat politique change pendant que vous tournez et, lorsque vous terminez votre film, les choses ne sont plus les mêmes que lorsque vous avez commencé. Si vous écrivez un article de journal, il est publié le lendemain. [...] Mais les réalisateurs qui choisissent une histoire contemporaine doivent prier pour que la situation qu'ils mettent en scène soit toujours d'actualité un an et demi plus tard. Dans le cas contraire, les gens pourraient dire que cela est de très mauvais goût $[. . .]^{3}$. (Phillips, 2001,107 , je traduis)

Les deux cinéastes vieillissants qui connaissaient, à l'époque de ces films, un relatif déclin de popularité, ont donc été plus ou moins dépassés par les événements ou se sont quelque peu égarés en ne souhaitant pas coller à l'actualité immédiate, Hitchcock revenant sur une crise réglée depuis des années déjà, et Wilder optant pour une intrigue contemporaine au tournage mais sans faire la moindre allusion au Mur de Berlin. Ces choix périlleux ainsi que les conditions de tournage complexes de Topaz et de One, Two, Three n'empêchèrent cependant ni Hitchcock ni Wilder de faire passer un certain nombre de messages politiques, en premier lieu grâce à une critique féroce de leurs personnages de Cubains, de Russes ou d'Allemands de l'Est.

\section{Une vision peu flatteuse des communistes}

Puisque Topaz, à la différence de One, Two, Three, n'est pas une comédie, les piques visant les communistes y sont moins explicites que dans le film de Wilder. Pourtant, bien que le long métrage d'Hitchcock reste une fiction, il comporte quelques aspects documentaires qui ne sont pas dénués de sous-entendus. Lorsque Rico Parra (John Vernon), un proche de Fidel Castro, séjourne à New York avec sa garde rapprochée, ils occupent tout un étage de l'hôtel Theresa de Harlem. Cet hôtel avait fermé ses portes au moment du tournage de Topaz, et il a donc été minutieusement reconstruit; Hitchcock tenait à cette dépense conséquente car le vrai Castro, venu prononcer l'un de ses discours aux Nations unies en 1960, était effectivement descendu dans cet établissement et y avait rencontré Malcolm X, Nehru, Nasser et Khrouchtchev. Mais ce qui fait réellement de l'hôtel Theresa un lieu historique et révolutionnaire, c'est qu'il a été l'un des premiers hôtels de New York à passer d'une clientèle et d'un personnel exclusivement blancs à des clients et employés blancs et noirs, un caractère multiracial parfaitement en accord avec les idéaux du régime révolutionnaire cubain.

Jusqu'ici, donc, Hitchcock se montre objectif, et son intrigue rappelle d'autant mieux l'histoire récente que l'acteur qui joue Rico Parra présente une troublante ressemblance avec Castro lui-même. Cependant, quelques détails furtifs indiquent que le réalisateur se souvient aussi des anecdotes qui ont émaillé la visite du Líder Máximo à New York neuf ans plus tôt. Il se trouve en effet que le vrai Castro et les autres membres de la délégation cubaine se sont d'abord installés dans un hôtel huppé situé 
sur Lexington Avenue; ils ont dû le quitter lorsqu'on leur aurait demandé 10000 dollars en liquide pour rembourser les dégâts qu'ils avaient causés : certains journaux de l'époque rapportent que Castro et ses hommes écrasaient leurs cigares sur les luxueux tapis et tuaient, plumaient et faisaient cuire des poulets dans leurs chambres. Lorsque Castro a voulu rentrer à Cuba au bout d'une dizaine de jours, il semblerait également que les autorités américaines soient allées jusqu'à lui confisquer son avion en guise de dédommagement, Khrouchtchev lui ayant alors prêté un appareil (Smith, 2016 ; Turkel, 2018).

13 Certes, dans son film, Hitchcock ne nous montre pas Rico Parra et son entourage aux prises avec des volatiles ; néanmoins, les scènes qui se déroulent à l'hôtel présentent les personnages de Cubains comme des rustres, et leur attitude décontractée frôle en fait le laisser-aller total, ce qui en dit long sur les capacités d'organisation que leur attribue Hitchcock. Le couloir de l'étage qu'ils occupent est bruyant et enfumé; certains hommes sont en compagnie de femmes, vraisemblablement des prostituées ; l'un d'eux, affalé contre un mur, se prépare à manger une orange dont il jette les pelures à même le sol - ce qui pourrait bien être un clin d'œil au sort que les vrais castristes auraient réservé à des poulets. Le garde du corps de Parra, Hernandez (Carlos Rivas), en vient même aux mains avec des visiteurs au milieu d'éclats de voix. La chambre à l'atmosphère poisseuse, qui sert aussi de bureau à Parra, ne donne pas une image plus positive des communistes cubains: il y règne un grand désordre et les restes de nourriture abondent; Parra, qui retrouve un contrat important sous une moitié de hamburger, tend tout naturellement le document maculé de graisse à son secrétaire pour qu'il lui en fasse deux copies; en outre, le révolutionnaire semble ne pouvoir se passer de son cigare et, s'il ne l'éteint pas sur un élément d'ameublement, il trouve en revanche normal de cracher sur la moquette de sa chambre. Dernier point, mais non le moindre, de nombreuses bouteilles d'alcool - pleines ou déjà vides - sont visibles aux quatre coins de la pièce ; d'ailleurs, à plusieurs reprises, Hitchcock cadre Parra en plan rapproché avec deux cannettes de bière en amorce : le réalisateur, en rendant Parra presque indissociable des bouteilles, qui occupent parfois plus de place que lui dans le cadre $^{4}$, insinue que ce personnage ne peut vivre et travailler sans boire de manière immodérée, et qu'il n'exerce aucun contrôle sur cette addiction. Lorsque René d'Arcy (George Skaff), l'ambassadeur de France à Washington, est présenté à son bureau, la sobriété du décor s'oppose diamétralement à l'environnement dans lequel travaille Parra; en outre, Hitchcock utilise également un plan rapproché pour cadrer René d'Arcy, mais ce sont cette fois des livres anciens à reliure de cuir, soigneusement ordonnés, qui sont visibles en amorce. A priori, Hitchcock prendrait donc le parti de montrer les communistes comme des personnages négligés aimant profiter de la vie et se livrant à des excès en tout genre pendant leurs journées de travail, et les capitalistes comme des personnages sérieux, organisés et attachés à la culture - ou, du moins, attachés à donner une telle image d'eux-mêmes.

Dans Topaz, Uribe (Don Randolph), l'assistant personnel de Parra, se fait acheter très facilement, une liasse de billets suffisant à transformer instantanément et miraculeusement un communiste convaincu en ardent capitaliste. Le scénario de One, Two, Three autorise le même type d'observation, mais la critique est assortie d'une note burlesque absente chez Hitchcock : les trois officiels russes, Peripetchikoff (Leon Askin), Mishkin (Peter Capell) et Borodenko (Ralf Wolter), bien qu'ils paraissent profondément anti-américains pendant la première moitié de l'intrigue, se laissent finalement aisément corrompre. Peripetchikoff va jusqu'à trahir la confiance de ceux qu'il appelait 
encore "camarades " quelques heures plus tôt, afin de passer à l'Ouest pour faire fortune dans les affaires en emmenant avec lui Ingeborg (Lilo Pulver), la sublime secrétaire de MacNamara (James Cagney). La façon dont Peripetchikoff compte devenir riche est totalement farfelue puisqu'il envisage d'échanger du fromage suisse contre de la choucroute qu'il vendra aux États-Unis, une fois transformée en guirlande de Noël plaquée argent. Bien qu'il tourne le dos à l'URSS, Peripetchikoff déclare que les Russes ont une longueur d'avance sur les Américains dans le domaine des missiles; mais là où le spectateur s'attend à des considérations d'ordre scientifique et technique, il explique qu'à Cap Canaveral, on dispose d'un bouton pour faire exploser un missile en cas de problème mais que les Russes, eux, disposent de deux boutons, le second étant destiné à l'ingénieur concepteur du missile défectueux. Le jeune Piffl (Horst Buchholz), Allemand de l'Est qui n'en finit pas de se révolter contre la décadence de l'Occident, assiste impuissant à la scène et lance à Peripetchikoff qu'il est pire encore que MacNamara, représentant de Coca-Cola à Berlin-Ouest, lequel, obsédé par la réussite, fait passer sa carrière avant sa famille. Piffl, au bord du désespoir, demande si le monde entier est corrompu, mais Peripetchikoff ne peut lui répondre... puisqu'il ne connaît pas tout le monde. Le mot de la fin revient à MacNamara : « une civilisation capable de produire le Taj Mahal, William Shakespeare et du dentifrice bicolore ne peut pas être foncièrement mauvaise » (je traduis) $)^{5}$.

Le sournois et très pragmatique MacNamara, héros du film qui voudrait coloniser le bloc de l'Est grâce à la célèbre boisson gazeuse, semble incarner à lui seul le capitalisme triomphant et pourrait ainsi être perçu comme le porte-parole de nombreux spectateurs américains d'origine du fait de ses jugements sans détour à propos des communistes et de ses réponses à certaines questions posées par les Russes : lorsque ces derniers lui demandent par exemple si, «plutôt que des dollars, [il] accepterait une tournée de trois semaines du Bolchoï », il rétorque, sans détour : "Par pitié, pas de culture : rien que du liquide » (je traduis) ${ }^{6}$. MacNamara parvient même, contre toute attente, à métamorphoser "Piffl le communiste bourru en parfait dandy capitaliste ", et cela en l'espace d'une matinée seulement (Michot, 2017, 164). Cette métamorphose est totale: Piffl adopte en quelques heures le mode de pensée et les manières d'un grand patron capitaliste, volant au passage une idée commerciale à MacNamara, ce qui montre qu'il a bien compris que pour réussir dans le monde des affaires, il fallait oublier l'égalité et la fraternité, et être dépourvu de morale et de scrupules. Mais c'est aussi et surtout par le biais des costumes que Wilder matérialise cette transformation réussie : Piffl, qui ne va pas chez le coiffeur et se refuse à porter des sous-vêtements, va subir une manucure et se voir offrir la garde-robe complète d'un gentleman. Dans la scène finale, il est présenté à sa belle-famille comme un comte, et il porte un costume trois pièces avec un œillet à la boutonnière, ainsi qu'un chapeau melon. MacNamara, roi de la manipulation, est pris à son propre piège car, pour Piffl, qui parvient sans peine à tromper son monde, il semble que l'habit fasse le moine et que cette nouvelle apparence aille de pair avec un revirement complet de ses opinions.

Un lien peut être établi entre One, Two, Three et Ninotchka (1939) dont Wilder a co-écrit le scénario pour Ernst Lubitsch, son maître absolu. Ninotchka, tourné à la veille du déclenchement de la Seconde Guerre mondiale, voit en effet la "conversion" de Soviétiques à la vie occidentale : les trois commissaires du peuple, Iranoff (Sig Ruman), Buljanoff (Felix Bressart) et Kopalski (Alexander Granach) - qui ne sont évidemment pas sans rappeler Peripetchikoff, Mishkin et Borodenko-, ne résistent pas longtemps aux joies de l'Ouest alors qu'ils sont installés dans la plus belle suite d'un luxueux hôtel 
parisien. Et c'est par un simple fondu enchaîné et grâce à des costumes, justement, que Lubitsch indique à ses spectateurs que ces personnages n'ont plus aucune foi en l'idéologie de leur pays: une casquette de prolétaire et deux toques en fourrure accrochées à un portemanteau s'effacent au profit d'un haut de forme et de deux chapeaux melon. Le chapeau melon est également symbole de promotion professionnelle dans The Apartment (La Garçonnière, Billy Wilder, 1960) comme il est une métaphore visuelle de l'ascension sociale pour le moins fulgurante de Piffl dans One, Two, Three.

17 Dans le film de Lubitsch, un autre chapeau, féminin cette fois, fait comprendre au public que Ninotchka est bien moins convaincue qu'elle ne l'affirme du déclin inexorable de l'Occident : une scène sans paroles, dans laquelle elle est seule, nous la montre retirant d'un meuble fermé à clé un chapeau ridicule ; elle le met sur sa tête et se regarde longuement dans un miroir ; or, elle avait publiquement méprisé ce chapeau en le voyant dans une vitrine. Que Ninotchka ait une apparence grotesque ne signifie pourtant pas que ses certitudes soient tournées en dérision, la critique du communisme comportant des nuances : comme le notent N.T. Binh et Christian Viviani, "jamais Ninotchka n'est ridiculisée. Ses idéaux mêmes ne sont pas traités par le mépris, contrairement aux habituels films anti-communistes hollywoodiens [...]; simplement, ils ne suffisent pas à bâtir un bonheur sans faille » $(1991,248)$. Par ailleurs, William Paul rappelle à juste titre que l'accessoire adopté par Ninotchka ne concrétise en aucun cas un revirement complet : « [cette] transformation physique liée à la décision de porter le chapeau ne matérialise en rien une conversion de la jeune femme au capitalisme (elle ne perd jamais ses convictions politiques, contrairement à la Ninotchka de Silk Stockings [La Belle de Moscou, Rouben Mamoulian, 1957]) ; cela représente plutôt l'acceptation de sa propre frivolité » $(1983,210 \text {, je traduis })^{7}$. Les messages politiques portés par les films hollywoodiens évoluent donc avec le temps : Ninotchka, sorti à la toute fin des années 30 , est surtout une critique du régime stalinien alors que Silk Stockings, qui date de la fin des années 50, donc de l'époque de Khrouchtchev, se pose en critique globale de l'idéologie communiste.

On notera en effet avec intérêt que, dans le remake de Mamoulian, Ninotchka (Cyd Charisse) troque ses vêtements sévères et tristes contre des tenues bien plus féminines - incluant les sous-vêtements en soie qui donnent au long métrage son titre original -, et qu'elle "capitule» par là-même, aux plans éthique et idéologique. Les tenues fonctionnelles sont remplacées par des froufrous que l'on ne revêt pas tant par besoin que par plaisir. Dans le Ninotchka de Lubitsch, le chapeau n'est d'ailleurs pas le seul vêtement à séduire la jeune Soviétique. Lorsqu'elle rentre dans son pays, elle explique à une amie, avec des étoiles dans les yeux, qu'en France, on change de toilette plusieurs fois dans la journée. Le superflu prend le pas sur le nécessaire, ce qui n'est pas pour déplaire à Iranoff, Buljanoff et Kopalski qui, en plus de leurs costumes trois pièces et de leurs couvre-chefs, ont opté pour des cannes. La tenue peut donc trahir des prises de position: ce sont des bas de soie, symboles de féminité par excellence, qui font abdiquer la Ninotchka de Mamoulian; de même, à la fin du film de Lubitsch, Ninotchka envisage de ne plus faire sécher sur le fil à linge commun le seul souvenir rapporté de Paris, une combinaison en soie, car celle-ci a déjà fait jaser toutes les femmes de l'immeuble qui accusent celle qui la porte d'être antirévolutionnaire.

Cette vision assez simpliste permet à des personnages de changer de tenue pour mieux tromper leur entourage. Dans l'épilogue de One, Two, Three, on l'a vu, les vêtements de 
Piffl ont un rôle au moins aussi important à jouer que le discours qu'il tient afin de convaincre les parents de sa toute récente épouse qu'il est le gendre idéal. Dans Topaz, Juanita la Cubaine (Karin Dor) ne rechigne pas à revêtir un treillis lorsqu'elle se tient à la tribune officielle à l'occasion d'un discours de Castro alors que dans sa vie de tous les jours, elle est particulièrement féminine. C'est ainsi que Devereaux (Frederick Stafford), son amant américain fraîchement arrivé sur l'île, prétend lui offrir des bas nylon sous les yeux de Parra, qui entretient lui-même une liaison avec elle et ignore son amour pour Devereaux ; la ruse fonctionne, Parra ne se doutant pas une seconde que Juanita trahit la cause qu'il défend et que le paquet contient en fait du matériel d'espionnage. Bien que la Ninotchka campée par Garbo succombe aux charmes de la vie parisienne sans renoncer à ses principes et à ses opinions politiques, elle finit par épouser un comte français; de la même manière, dans One, Two, Three, Piffl l'Allemand de l'Est se marie à une héritière américaine. Quant aux trois commissaires des deux films, ils abandonnent également la Russie pour se lancer avec succès dans les affaires, en n'hésitant pas à se duper les uns les autres. Selon Michel Ciment, le monde est, pour Wilder, «le théâtre d'une lutte sans merci où les communistes sont vus comme des capitalistes en puissance qui sont forcés de réprimer leurs instincts » (Ciment, 2015, 153).

20 Outre ces références à d'autres œuvres de fiction, Wilder, tout comme Hitchcock, fait allusion à une anecdote célèbre dans le scénario de One, Two, Three. Lorsque MacNamara, qui cherche à soudoyer les trois Russes, leur rend visite à leur hôtel de Berlin-Est - un établissement délabré aussi peu engageant que celui qui accueille les Cubains dans Topaz-, Ingeborg se met à danser de manière très suggestive et «Borodenko tape sur la table avec sa chaussure - non pour protester comme Khrouchtchev l'aurait fait à l'ONU mais, au contraire, pour [...] montrer son contentement. C'est d'ailleurs à ce moment-là que l'immense portrait de Khrouchtchev accroché au mur de la salle glisse de son cadre et révèle un portrait de Staline " (Michot, 2017, 162). En effet,

un an avant la sortie de One, Two, Three, lors de l'Assemblée générale de l'ONU du 12 octobre 1960, Nikita Khrouchtchev a été filmé tapant violemment du poing sur son pupitre pour marquer son opposition au délégué philippin qui critiquait la tutelle de Moscou sur les pays de l'Est. Des journalistes présents ont rapporté que Khrouchtchev était allé jusqu'à utiliser sa chaussure pour frapper la table, mais aucune caméra ni aucun photographe n'a immortalisé la scène, ce qui tendrait à prouver qu'elle n'a jamais existé. Toutefois, elle reste gravée dans la mémoire ou la mythologie collective comme un incident marquant de la guerre froide (Michot, 2017, 162),

au même titre que le comportement peu civilisé qu'auraient eu Castro et ceux qui l'accompagnaient, le mois précédent dans un hôtel new-yorkais.

Une autre allusion du même style peut être décelée dans les dialogues. La jeune Scarlett (Pamela Tiffin), fille du grand patron d'Atlanta, cause bien des soucis à MacNamara qui est censé veiller sur elle alors qu'elle séjourne à Berlin. Quand Ingeborg demande à MacNamara si les écoles ne vont pas rouvrir bientôt, celui-ci lui répond : «En Géorgie ? On ne peut être sûr de rien " (je traduis) ${ }^{8}$. Cette réplique est une référence à l'actualité de janvier 1961 lorsqu'après une longue bataille judiciaire, deux étudiants noirs ont été autorisés à s'inscrire à l'Université de Géorgie qui n'acceptait jusque-là que des Blancs. Des émeutes ont tout de même éclaté car certains extrémistes contestaient une telle décision, mais ces débordements n'ont pas empêché la déségrégation définitive du 
campus, événement pour le moins révolutionnaire dans cet État du Sud profond (Besel, 2017 ; « Desegregation of UGA », 2013).

Cette remarque de MacNamara, qui pourrait passer inaperçue au milieu d'un flot de dialogues au débit soutenu, pointe du doigt le problème du racisme et suggère que les USA sont loin d'être un pays parfait. Par conséquent, la repartie, l'assurance voire l'arrogance de MacNamara, personnage qui domine l'intrigue, sont à double tranchant ; de la même manière, les espions occidentaux - loyaux ou traîtres - de Topaz ainsi que la construction du scénario d'Hitchcock révèlent une posture ambivalente. Les deux réalisateurs, loin d'être manichéens, n'hésitent pas à écorcher l'image des États-Unis alors dominante en Occident, en dépassant le mythe d'un pays érigé comme modèle au plan économique mais aussi idéologique pour avoir sauvé l'Europe de la barbarie nazie.

\section{Des capitalistes vraiment épargnés ?}

Certes, avec One, Two, Three, Wilder et Diamond ne se privent pas de ridiculiser les communistes, mais la réalité censée être représentée est particulièrement déformée, et les personnages en question, qui semblent tous aisément manipulables tant ils sont idiots, sont peut-être trop caricaturaux pour rester crédibles. Peripetchikoff, Mishkin et Borodenko, par exemple, vont se plaindre aux Suisses qui ont osé leur vendre du fromage plein de trous; en outre, MacNamara se moque des trois Russes en leur rappelant que personne ne veut boire de leur Kremlin-Cola, pas même les Albanais qui s'en servent comme antiparasitaire pour les moutons. Piffl, quant à lui, ne connaît pas le couteau et la fourchette et saisit sa nourriture à pleines mains; lorsqu'il se fait arrêter par la police est-allemande, c'est parce qu'il transporte à son insu un coucou d'où sort toutes les heures l'Oncle Sam agitant la bannière étoilée au son de Yankee Doodle; enfin, le jeune homme méprise tant la "culture » occidentale qu'il suffit à la Stasi de lui faire écouter en boucle Itsy Bitsy Teenie Weenie Yellow Polkadot Bikini pour qu'il avoue être un espion américain.

Les conditions de vie derrière le rideau de fer sont également tournées en dérision: c'est avec le plus grand sérieux que Piffl dit à Scarlett de se hâter car le train de 7h pour Moscou part à 8 h15 précises; et il lui promet qu'en Russie, elle prendra tous les jours son petit déjeuner au lit, ainsi que son déjeuner et son dîner... car leur logement n'aura ni table ni chaises. Concernant l'absence de confort, la promiscuité et les salles de bains communes (également représentées dans une des dernières séquences de Ninotchka), Wilder aimait à raconter une anecdote. Pendant le tournage de One, Two, Three, The Apartment a été projeté dans un établissement culturel de Berlin-Est, et il a été très applaudi par les invités communistes qui y voyaient une "critique de l'univers capitaliste de la consommation et du travail où tout le monde doit se vendre ». Le réalisateur, invité à donner une petite conférence, a déclaré que ce qu'il avait montré dans le film (un employé qui, en échange d'une promotion, prête son appartement à ses supérieurs pour des aventures extra-conjugales) pouvait se passer n'importe où ailleurs. "Il n'y a qu'une ville au monde où cela ne peut pas arriver, c'est Moscou. [Applaudissements flattés de l'auditoire.] Cela ne peut pas se passer à Moscou pour une raison simple, c'est que Lemmon n'aurait pas pu y louer son appartement. Parce qu'il l'aurait partagé avec trois autres familles. [Silence accablé.] 》 (Wilder et Karasek, 1993, 415). 
C'est une impressionnante parade militaire sur la place Rouge qui ouvre Topaz. La première séquence de One, Two, Three contient le même type de cliché : elle présente en effet les habitants de Berlin-Est qui s'adonnent à ce qui est, selon MacNamara, leur activité favorite : défiler en brandissant des portraits de leaders communistes ainsi que des banderoles aux slogans anti-capitalistes, chantant L'Internationale à tue-tête et laissant s'envoler des dizaines de ballons portant l'inscription "Yankee go home ». Le cortège passe au milieu des ruines, "comme si la reconstruction pouvait attendre " (Michot, 2017, 166), et les bâtiments détruits s'inscrivent en porte-à-faux avec l'ordre apparent du défilé. La musique diégétique offre également une contradiction ironique avec l'image (Stam, 2005, 20). L'Internationale est un morceau préexistant qui a une portée assez universelle pour que les spectateurs puissent l'identifier ; en outre, même si l'hymne est ici chanté en allemand, la teneur de ses paroles est connue de tous. Pourtant, bien que les personnages soient en mouvement, c'est une impression d'immobilisme qui domine : alors que ce chant révolutionnaire appelle à aller de l'avant et à mener une lutte sociale, l'image suggère que les habitants de Berlin-Est se contentent de protester et qu'ils emploient leur énergie à défiler inlassablement, mais sans réellement agir pour que leur situation s'améliore.

MacNamara explique ensuite, en voix-off, que les choses sont bien différentes dans le secteur ouest de la ville, placé sous protection alliée, et dont les habitants sont en mesure de profiter de "tous les bienfaits de la démocratie ». Au même moment, la caméra, qui effectue un panoramique, s'arrête sur un immense panneau publicitaire qui montre une jeune femme en bikini vantant les mérites du Coca-Cola. Selon Neil Sinyard et Adrian Turner, le message est clair : la reconstruction de cette partie de la ville se fait grâce aux capitaux américains, et l'on pourrait donc penser que Wilder glorifie l'esprit d'initiative de son pays d'adoption. Pourtant, les deux auteurs ajoutent que cette alliance de la diplomatie et du dollar est inévitablement porteuse de désintégration morale (Sinyard et Turner, 1979, 105). Et en effet, il n'est pas anodin que Wilder associe l'affiche publicitaire en question au terme si noble de "démocratie »: l'image vient à nouveau démentir ce qu'énonce MacNamara avec tant de conviction. Dès les premières minutes $\mathrm{du}$ film, donc, le réalisateur fait preuve d'ironie et insinue que lorsqu'il est question de propagande, l'Amérique surpasse amplement le bloc de l'Est ; il ne s'agit pas d'une ironie d'ordre dramatique qui influerait sur le déroulement de l'action et aurait des conséquences négatives sur les personnages (MacDowell, 2016, 36-38) mais plutôt d'une façon, pour Wilder, d'établir une distance avec son protagoniste et de le décrédibiliser en exposant d'emblée la vraie nature de ses "idéaux». Car bien que l'intrigue et les personnages de One, Two, Three soient totalement fictifs, les cadres géographique et idéologique de l'action correspondent bien à une réalité contemporaine au tournage. C'est, comme souvent, par un humour incisif que le réalisateur fait passer ses prises de position. Le ton est donné, d'autant plus que l'on a ici un procédé de «double ironie » puisque MacNamara lui-même, qui fait office de narrateur dans cet incipit, est ironique vis-à-vis des communistes qui vivent à Berlin-Est.

MacNamara se targue d'ailleurs, par le biais de Coca-Cola, d'être sur le point de réussir à conquérir Moscou, alors que Napoléon et Hitler ont échoué avant lui. Mais est-ce vraiment une fierté que de faire « mieux » qu'Hitler? L'épouse de MacNamara (Arlene Francis) est, comme le note Kevin Lally, le seul personnage non caricatural du film $(1996,318)$ et aussi le plus lucide, et il n'y a donc rien d'étonnant à ce qu'elle appelle 
régulièrement son mari «Mein Führer ». La blague n'en est peut-être pas vraiment une, et ce petit détail suffit à mettre à mal les valeurs américaines incarnées par MacNamara. Narrateur et figure dominante du film dont l'énergie débordante semble toujours être employée à bon escient, MacNamara subira un échec cuisant dans les dernières minutes de l'intrigue en étant évincé par celui que le public croyait dépourvu d'intelligence et de discernement puisque communiste - le jeu outré de Horst Buchholz accentuant de fait l'impression que Piffl est borné et complètement idiot.

Le film d'Hitchcock contient des sous-entendus similaires mais le réalisateur préférant les images aux dialogues, ces indices sont surtout visuels. Donald Spoto affirme que, dans Topaz, "plusieurs indications subtiles montrent qu'Hitchcock rend, de manière voilée, les États-Unis responsables des souffrances et des assassinats que subissent les personnages de son film. [...] Deux lampes placées au-dessus de la tête de l'agent américain McKittreck (Edmon Ryan) lui dessinent des cornes diaboliques [...] (Spoto, 1986, 287-288). Par ailleurs, lorsqu'on se penche sur la chronologie fine du scénario, on constate qu'Hitchcock prend des libertés quant aux événements décrits par Leon Uris, un choix qui est lourd de conséquences: dans le roman, lorsque André Devereaux, l'agent français qui travaille pour les Américains, se rend à Cuba, il en rapporte les preuves de la présence de missiles soviétiques, ce qui permet à l'administration Kennedy d'agir ; dans le film, en revanche, la visite de Devereaux à Cuba est bien plus tardive : à bord de l'avion qui l'emmène dans l'île, Devereaux lit le New York Times daté du 22 octobre 1962, dont les gros titres indiquent que les États-Unis sont déjà au courant de ce que les Soviétiques préparent à Cuba, une intervention télévisée de Kennedy étant imminente. Cela signifie que la mission de Devereaux n'a aucune utilité et, surtout, que sa maîtresse Juanita, qui est à la tête d'un réseau anti-castriste, va mourir pour rien, de même que les Mendoza (Anna Navarro et Lewis Charles), torturés à mort pour avoir volé des photos de manœuvres militaires (Walker, 2005, 298-299). La dénonciation de la guerre froide par Hitchcock est donc globale, et il incrimine le bloc communiste autant que le bloc capitaliste. Le réalisateur faisait déjà de même dans Torn Curtain, un film qui contient son lot de personnages tristes prêts à tout pour passer à l'Ouest ou qui ont la nostalgie des États-Unis. Dans ce film, le bloc communiste baigne dans un climat de désolation, de suspicion permanente et de délation. On ne parvient pourtant pas à oublier que le scientifique américain campé par Paul Newman, le Professeur Armstrong, va voler derrière le rideau de fer une formule qu'il n'a pas su trouver lui-même, et ce afin de donner un nouvel élan à sa carrière. Si son entreprise est couronnée de succès, c'est au prix de la mort d'un homme, Gromek (Wolfgang Kieling), qui ne faisait que son travail et qu'il a assassiné de ses propres mains sans paraître en éprouver le moindre remords. Rien n'est dit ni explicitement montré concernant le sort réservé à la fermière (Carolyn Conwell) qui a aidé Armstrong à se débarrasser de Gromek, ou celui de la comtesse Kuchinska (Lila Kedrova) qui comptait sur le Professeur pour pouvoir émigrer aux États-Unis et qui s'est interposée pour lui sauver la vie. Toute l'organisation permettant à des réfugiés de rejoindre l'Ouest est mise en péril à cause d'Armstrong, qui n'agit pas au service de la liberté ou d'une idéologie, mais bien dans l'intérêt de sa propre personne. Il n'est d'ailleurs pas étonnant que l'épilogue du film d'Hitchcock mentionne une "valeur" proprement matérialiste : lorsqu'il arrive en Suède, Armstrong prononce ces mots : « nous avons de l'argent ». du public, comme Cary Grant ou James Stewart, qui ont tous deux tourné plusieurs 
films avec Hitchcock. Les actes d'Armstrong dans Torn Curtain paraîtraient peut-être moins légitimes au spectateur si un acteur moins charismatique (et aux yeux moins bleus ?) incarnait ce personnage. Et justement, aucune grande star américaine ne figure au casting de Topaz, ce qui joue un rôle important dans la perception des camps capitaliste et communiste. Les personnages masculins occidentaux paraissent interchangeables : ils affichent la même expression sérieuse voire solennelle, sont vêtus de costumes cravates, et John Forsythe, interprète de l'agent de la CIA Michael Nordstrom, présente une réelle ressemblance avec Frederick Stafford, qui joue l'agent français. Claude Jade et Michel Subor, quant à eux, incarnent la fille modèle et le gendre idéal des Devereaux. Du côté communiste, les personnages sont nettement plus hauts en couleurs: le Russe Boris Kusenov (Per-Axel Arosenius) est énigmatique et imprévisible; Hernandez, le garde du corps cubain, ne passe pas inaperçu avec sa chevelure et sa barbe rousses; le chétif Uribe, assistant de Parra, détonne également par sa nervosité permanente et ses petites lunettes rondes qui ressemblent à deux loupes; enfin, la blonde Nicole Devereaux (Dany Robin) ne voit son mari revenir vers elle que parce que la sublime et piquante Juanita a trouvé la mort. À ce propos, le public ne peut qu'éprouver de l'empathie pour Parra, détruit lorsqu'il apprend la double trahison de Juanita, et contraint de tuer cette femme qu'il aime afin de lui épargner la torture. Le passage, techniquement très abouti, et qui nous rappelle qu'Hitchcock se plaisait à filmer ses scènes d'amour comme des scènes de meurtre et inversement, contient un lent travelling circulaire puis une plongée à la verticale lorsque Juanita, les yeux grands ouverts, s'effondre sur le sol carrelé, sa longue robe violette s'ouvrant comme une corolle. La réaction de Devereaux à l'annonce du décès de Juanita quelques soupirs résignés et un air sombre qui ne contraste guère avec l'expression de son visage dans le reste du film - peine, au contraire, à susciter une identification de la part du spectateur.

L'épilogue de Topaz, comme celui de One, Two, Three, confirme la position des deux films. L'originalité de Topaz est qu'un certain nombre de fins ont été envisagées, et trois tournées. Celle qui avait la préférence d'Hitchcock - mais pas celle du public des previews - consistait en un duel au revolver dans un stade désert entre Jacques Granville (Michel Piccoli), le haut fonctionnaire français à la solde des communistes, et André Devereaux. Granville était en fait abattu par un tireur isolé payé par les Russes. Une autre fin, qui est celle de la version longue du film diffusée de nos jours dans les pays anglophones, montre Devereaux et sa femme Nicole - qui, quelques heures auparavant, était encore la maîtresse de Granville - quitter Paris pour rentrer à Washington à bord d'un avion de la Pan American, alors que Granville embarque au même moment sur un vol Aeroflot pour Moscou et salue le couple avec un large sourire. D'après Laurent Bourdon, il a fallu renoncer à cet épilogue pour ne pas froisser le gouvernement français qui «ne voulait pas laisser à croire qu'un haut fonctionnaire ayant trahi son pays pouvait, à si bon compte, quitter le sol de la République pour rejoindre ses chers camarades " (Bourdon, 2007, 314). La fin qui a été adoptée à la sortie du film pour tous les pays et qui est toujours celle des versions diffusées en France, suggère que Granville, apprenant qu'il est démasqué, préfère se suicider. Il n'y a donc plus d'espion russe infiltré au sein même du gouvernement français et la crise des missiles est terminée, comme l'annonce une nouvelle une de journal ; on voit toutefois défiler à l'écran, en surimpression et fondu-enchaîné, les scènes d'assassinat ou de torture qui ont émaillé l'intrigue et représentent le prix qu'il a fallu payer. Selon Spoto, «le cynisme moral d'Hitchcock, sa profonde méfiance à l'égard de la politique, son mépris du grand 
commerce international n'ont jamais été aussi évidents que dans ce film » (Spoto, 1986, 289).

31 Wilder est-il aussi désenchanté ? Sans doute, mais le caractère éminemment burlesque de One, Two, Three gomme quelque peu la férocité de la critique idéologique : alors que la voiture transportant MacNamara et le jeune couple file à toute allure vers l'aéroport pour accueillir les parents de Scarlett, un artisan, penché par la fenêtre, peint avec une grande précision, et donc à l'envers, les armoiries de Piffl, devenu le comte Otto von Droste-Schattenburg. On ne peut toutefois nier que MacNamara réalise un véritable exploit lorsqu'il parvient à transformer Piffl en gendre parfait aux yeux de Wendell P. Hazeltine (Howard St. John), père de Scarlett, grand patron de Coca-Cola, et conservateur de l'Amérique profonde qui arrive presque sans prévenir d'Atlanta. Mais cette étonnante métamorphose a une conséquence pour le moins inattendue : Piffl est si convaincant que son beau-père, qui ne le connaît que depuis quelques minutes, décide de lui confier un poste important à Londres, celui que MacNamara convoitait depuis des années. La morale de l'histoire est bien que «[...] les Américains sont beaucoup plus efficaces que les Soviétiques pour coloniser les cerveaux» (Simsolo, 2008, 70), l'ironie étant que l'efficacité de MacNamara se retourne contre lui.

De même, dans Topaz, le personnage qui s'en sort le mieux est sans nul doute Boris Kusenov: au début de l'intrigue, il passe à l'Ouest avec sa famille, mais se montre méfiant et condescendant vis-à-vis des Américains; plus tard, on le retrouve dans le confortable manoir mis à sa disposition, l'air satisfait, fumant un bon cigare (cubain ?) et se faisant servir par des domestiques. Avant de s'éclipser pour aller profiter du jardin, laissant Américains et Français démêler seuls une affaire compliquée, il conseille à Devereaux de l'imiter, et de penser à son intérêt personnel plutôt qu'à son devoir envers son gouvernement. Cette dernière apparition de Kusenov, comme la dernière apparition de Piffl dans le film de Wilder, est la preuve ultime que les communistes battent aisément les capitalistes à leur propre jeu.

\section{Conclusion}

À la mort d'Hitchcock, le journal soviétique Literaturnaya Gazeta s'est montré très critique envers le réalisateur, dont l'œuvre, selon lui, faisait l'apologie du vice et de la cruauté, et était le reflet de la décadence de la société occidentale. Les journalistes ciblaient en particulier Torn Curtain et Topaz pour leur caractère anti-russe et la façon dont leur intrigue "brouillait les frontières entre ce qui était moral et immoral " (Humphries, 1987, 167-168; je traduis) 9 . Les jeunes Américains pacifistes qui ont découvert Topaz en 1969 ont vu dans ce film une dénonciation des régimes communistes et une façon de considérer les Russes et les Cubains comme des menaces à l'ordre mondial, ce qui leur a évidemment déplu. Mais Hitchcock, bien qu'il semble mettre en scène une œuvre conventionnelle en accord avec les codes et l'état d'esprit hollywoodiens, n'est pas si conservateur dans la façon dont il traite cette histoire polémique. Uribe et Juanita sont exécutés par Parra, et les Mendoza connaissent une lente agonie dans une geôle cubaine seulement parce que Devereaux, un Français au service des Américains, s'est servi d'eux sans le moindre scrupule. Si Devereaux, lui, n'a pas de sang sur les mains, en aucun cas le scénario ne l'érige en exemple, ce qui souligne à quel point les valeurs associées aux pays occidentaux suscitent la méfiance d'Hitchcock. 

Ciment au tout début des années 80 à propos de Ninotchka et de One, Two, Three, il allait jusqu'à déclarer qu'il «ador[ait] irriter tout le monde ", que ce soient les communistes ou les capitalistes (Tresgot et Ciment, 1998, 51'10"). Dans One, Two, Three, l'humour est corrosif et les régimes en place derrière le rideau de fer sont effectivement visés par la satire, ce que la critique de gauche a reproché à Wilder à la sortie du film (Simsolo, 2008, 69) ; par ailleurs, le bloc occidental étant lui aussi malmené, la presse outreAtlantique a immédiatement accusé le réalisateur d'être "anti-américain » (Phillips, 2010, 250). Il est vrai que MacNamara tourne en dérision le totalitarisme tout en s'échinant à faire boire du Coca-Cola à tous ceux qui ne le font pas déjà - une curieuse façon d'envisager la démocratie. On note tout de même avec intérêt que Wilder et Diamond, en dépit des exagérations de leur script, se montrent assez visionnaires : au moment de la chute du Mur, l'un des premiers plaisirs des habitants de Berlin-Est a été de goûter à la liberté... et au Coca-Cola (Canas, 2009) ; de manière moins réjouissante, au début du Xxi ${ }^{e}$ siècle, l'armée américaine a utilisé la musique à des fins de déstabilisation psychologique à Guantánamo, ce qui fait tristement écho à la scène de torture de Piffl par la Stasi dans le film.

Alors, qui sort gagnant des deux films? Certainement pas leurs réalisateurs, bien que One, Two, Three, à sa ressortie au moment de la réunification, ait connu un véritable succès populaire, particulièrement auprès des spectateurs allemands. Bien plus tôt, en 1967, soit six ans après la sortie originale du film de Wilder, une courte scène du James Bond parodique Casino Royale (John Huston, Kenneth Hughes, Val Guest, Robert Parrish et Joseph McGrath) prouve que le public était désormais prêt à accepter les plaisanteries en lien avec la guerre froide - une sorte de revanche pour Wilder qui avait participé à ce projet en suggérant quelques idées aux scénaristes : une plongée présente une rue animée de Berlin-Ouest avec ses bars et cabarets, puis la caméra prend de la hauteur pour filmer l'autre côté du Mur, triste et désert (si l'on exclut la présence de militaires) et baigné de lumière rouge, alors que la musique de fosse pop et jazzy de Burt Bacharach a fait place à quelques mesures d'Otchi Tchornye jouées à la mandoline avec une grande mélancolie.

'épilogue de Topaz d'Hitchcock, quelle qu'en soit la version, sonne plutôt comme un match nul entre capitalistes et communistes, la guerre froide devant se poursuivre pendant de longues années. En outre, Juanita la Cubaine, en s'opposant au gouvernement issu de la lutte menée par son défunt mari, confirme que la révolution, plus qu'une rupture, est bien un éternel recommencement. Cette idée de retour au point de départ est à l'image de l'apparition rituelle d'Hitchcock, l'une des plus facétieuses de toutes : assis dans un fauteuil roulant poussé par une infirmière, il se lève soudain sans la moindre difficulté et se met à marcher en levant bien haut le genou; ce caméo peut être vu comme un avertissement à destination des spectateurs il ne faut pas se fier aux apparences - ou comme une façon pour Hitchcock de montrer qu'en 1969, il est encore dans la course, bien qu'il n'ait pas l'âme d'un révolutionnaire. 


\section{BIBLIOGRAPHIE}

BESEL, Peter. « University of Georgia Desegregation Riot (1961) ». BlackPast.org. 2017. http:// www.blackpast.org/aah/university-georgia-desegregation-riot-1961. Page consultée le 30 mai 2018.

BINH, N.T., et Christian VIVIANI. Lubitsch. Paris : Rivages, Rivages Cinéma, 1991.

BOURDON, Laurent. Dictionnaire Hitchcock. Paris : Larousse, 2007.

CANAS, Bastien. «Chute du mur de Berlin : une journée historique pour Coca-Cola », Coca-Cola Web. 9 novembre 2009. https://cocacolaweb.fr/chute-du-mur-de-berlin-une-journee-historiquepour-coca-cola/. Page consultée le $1^{\text {er }}$ juin 2018.

CIMENT, Michel. Les Conquérants d'un nouveau monde : Essais sur le cinéma hollywoodien. 1981. Paris : Gallimard, Folio Essais, 2015.

« Desegregation of UGA ». Today in Georgia History, 2013, http://www.todayingeorgiahistory.org/ content/desegregation-uga. Page consultée le 30 mai 2018.

HARK, Ina R. « Keeping Your Amateur Standing: Audience Participation and Good Citizenship in Hitchcock's Political Films ». Cinema Journal, vol. 29, nº 2, 1990, p. 8-22.

HUMPHRIES, Patrick. The Films of Alfred Hitchcock. Londres : Bison Books, 1987.

LALLY, Kevin. Wilder Times: The Life of Billy Wilder. New York : Henry Holt \& Co., 1996.

MACDOWELL, James. Irony in Film. Londres : Palgrave Macmillan, Palgrave Close Readings in Film and Television, 2016.

MADSEN, Axel. Billy Wilder. Bloomington : Indiana University Press, Cinema One, 1969.

MICHOT, Julie. Billy Wilder et la musique d'écran : filmer l'invisible. Reims : Éditions et Presses Universitaires de Reims, Studia Remensia, 2017.

PAUL, William. Ernst Lubitsch's American Comedy. New York : Columbia University Press, 1983.

PHILLIPS, Gene D. « Billy Wilder ». Billy Wilder: Interviews. Dir. Robert Horton. Jackson : University Press of Mississippi, Conversations with Filmmakers, 2001, p. 99-109.

---. Some Like It Wilder: The Life and Controversial Films of Billy Wilder. Lexington : University Press of Kentucky, Screen Classics, 2010.

SIMSOLO, Noël. Billy Wilder. Paris : Cahiers du cinéma/Le Monde, Grands Cinéastes, 2008.

SINYARD, Neil, et Adrian TURNER. Journey Down Sunset Boulevard: The Films of Billy Wilder. Ryde : BCW Publishing Limited, 1979.

SMITH, David. "Fidel Castro in the US: cars, cigars and a meeting with Malcolm X », The Guardian. 27 november 2016. https://www.theguardian.com/world/2016/nov/27/fidel-castro-new-yorkmalcolm-x. Page consultée le 29 mai 2018.

SPOTO, Donald. L'Art d'Alfred Hitchcock : Hitchcock, 50 ans de films. Paris : Edilig, 1986.

STAM, Robert, dir. New Vocabularies in Film Semiotics. New York : Routledge, 2005.

TRESGOT, Annie, et Michel CIMENT. Portrait of a « $60 \%$ Perfect » Man: Billy Wilder, Action Films, 1980. DVD. Agat Films \& Cie, 1998.

TRUFFAUT, François. Hitchcock/Truffaut, Édition définitive. Paris : Gallimard, 1997. 
TURKEL, Stan. « Hotel Theresa: The Waldorf of Harlem ». The Most Famous Hotels in the World, 2018. https://famoushotels.org/news/hotel-theresa-the-waldorf-of-harlem. Page consultée le 29 mai 2018.

URIS, Leon. Topaz: A Novel. New York : McGraw-Hill, 1967.

WALKER, Michael. Hitchcock's Motifs. Amsterdam : Amsterdam University Press, Film Culture in Transition, 2005.

WILDER, Billy, et Helmut KARASEK. «Et tout le reste est folie » : Mémoires. Paris : Robert Laffont, Vécu, 1993.

WOOD, Thomas. « In Wilder's Wild West ». Billy Wilder: Interviews. Dir. Robert Horton. Jackson : University Press of Mississippi, Conversations with Filmmakers, 2001, p. 35-37.

\section{Filmographie}

Ace in the Hole. Réal. Billy Wilder. Scénario de Billy Wilder, Lesser Samuels et Walter Newman. Avec Kirk Douglas (Chuck Tatum), Jan Sterling (Lorraine Minosa), Robert Arthur (Herbie Cook) et Porter Hall (Jacob Q. Boot). Paramount Pictures, 1951. DVD. Eureka, 2014.

Alice's Restaurant. Réal. Arthur Penn. Scénario de Venable Herndon et Arthur Penn. Avec Arlo Guthrie (Arlo), Patricia Quinn (Alice), James Broderick (Ray) et Pete Seeger (Pete Seeger). Elkins Entertainment / Florin, 1969. DVD. Twentieth Century Fox, 2003.

Blow-Up. Réal. Michelangelo Antonioni. Scénario de Michelangelo Antonioni et Tonino Guerra. Avec Vanessa Redgrave (Jane), Sarah Miles (Patricia), David Hemmings (Thomas) et John Castle (Bill). MGM / Premier Productions, 1966. DVD. Warner Bros., 2004.

Casino Royale. Réal. John Huston, Kenneth Hughes, Val Guest, Robert Parrish et Joseph McGrath. Scénario de Wolf Mankowitz, John Law et Michael Sayers. Avec Peter Sellers (Evelyn Tremble), Ursula Andress (Vesper Lynd), David Niven (Sir James Bond) et Orson Welles (Le Chiffre). Famous Artists Productions, 1967. DVD. MGM / United Artists, 2001.

Easy Rider. Réal. Dennis Hopper. Scénario de Peter Fonda, Dennis Hopper et Terry Southern. Avec Peter Fonda (Wyatt), Dennis Hopper (Billy), Antonio Mendoza (Jesus) et Phil Spector (Connection). Pando Company / Raybert Productions, 1969. DVD. Sony Pictures, 1999.

Fedora. Réal. Billy Wilder. Scénario de Billy Wilder et I.A.L. Diamond. Avec William Holden (Barry Detweiler), Marthe Keller (Fedora), Hildegard Knef (The Countess) et José Ferrer (Doctor Vando). Bavaria Atelier / Lorimar / NF Geria Filmgesellshaft / SFP, 1978. DVD. Carlotta Films, 2014.

Foreign Correspondent. Réal. Alfred Hitchcock. Scénario de Charles Bennett et Joan Harrison. Avec Joel McCrea (John Jones), Laraine Day (Carol Fisher), Herbert Marshall (Stephen Fisher) et George Sanders (Scott Ffolliott). Walter Wanger Productions, 1940. DVD. Studiocanal, 2012.

Lifeboat. Réal. Alfred Hitchcock. Scénario de Jo Swerling. Avec Tallulah Bankhead (Connie Porter), William Bendix (Gus Smith), Walter Slezak (Willi) et Mary Anderson (Alice MacKenzie). Twentieth Century Fox, 1943. DVD. Twentieth Century Fox, 2006.

Midnight Cowboy. Réal. John Schlesinger. Scénario de Waldo Salt. Avec Dustin Hoffman (Ratso), Jon Voight (Joe Buck), Sylvia Miles (Cass) et John McGiver (Mr. O’Daniel). Jerome Hellman Productions, 1969. DVD. MGM / United Artists, 1999. 
Ninotchka. Réal. Ernst Lubitsch. Scénario de Charles Brackett, Billy Wilder et Walter Reisch. Avec Greta Garbo (Ninotchka), Melvyn Douglas (Count Leon d'Algout), Ina Claire (Grand Duchess Swana) and Bela Lugosi (Commissar Razinin). MGM, 1939. DVD. Warner Bros., 2006.

Notorious. Réal. Alfred Hitchcock. Scénario de Ben Hecht. Avec Cary Grant (Devlin), Ingrid Bergman (Alicia Huberman), Claude Rains (Alexander Sebastian) et Louis Calhern (Paul Prescott). RKO Radio Pictures, 1946. DVD. Carlotta Films, 2018.

One, Two, Three. Réal. Billy Wilder. Scénario de Billy Wilder et I.A.L. Diamond. Avec James Cagney (C.R. MacNamara), Horst Buchholz (Otto Ludwig Piffl), Pamela Tiffin (Scarlett Hazeltine) et Arlene Francis (Phyllis MacNamara). Bavaria Film / Pyramid Productions / The Mirisch Corporation, 1961. DVD. Rimini Éditions, 2019.

Saboteur. Réal. Alfred Hitchcock. Scénario de Peter Viertel, Joan Harrison et Dorothy Parker. Avec Priscilla Lane (Patricia Martin), Robert Cummings (Barry Kane), Otto Kruger (Charles Tobin) et Norman Lloyd (Frank Fry). Frank Lloyd Productions / Universal Pictures, 1942. DVD. Universal Pictures France, 2017.

Sabrina. Réal. Billy Wilder. Scénario de Billy Wilder, Samuel Taylor et Ernest Lehman. Avec Humphrey Bogart (Linus Larrabee), Audrey Hepburn (Sabrina Fairchild), William Holden (David Larrabee) et John Williams (Thomas Fairchild). Paramount Pictures, 1954. DVD. Paramount Pictures, 2009.

Silk Stockings. Réal. Rouben Mamoulian. Scénario de Leonard Gershe et Leonard Spigelgass. Avec Fred Astaire (Steve Canfield), Cyd Charisse (Ninotchka Yoschenko), Janis Paige (Peggy Dayton) et Peter Lorre (Brankov). MGM, 1957. DVD. Warner Bros., 2006.

Sunset Boulevard. Réal. Billy Wilder. Scénario de Charles Brackett, Billy Wilder et D.M. Marshman Jr. Avec William Holden (Joe Gillis), Gloria Swanson (Norma Desmond), Erich von Stroheim (Max von Mayerling) et Nancy Olson (Betty Schaefer). Paramount Pictures, 1950. DVD. Paramount Pictures, 2003.

The Apartment. Réal. Billy Wilder. Scénario de Billy Wilder et I.A.L. Diamond. Avec Jack Lemmon (C.C. Baxter), Shirley MacLaine (Fran Kubelik), Fred MacMurray (Jeff D. Sheldrake) et Ray Walston (Joe Dobisch). The Mirisch Corporation, 1960. DVD. MGM / United Artists, 2001.

The Lost Weekend. Réal. Billy Wilder. Scénario de Charles Brackett et Billy Wilder. Avec Ray Milland (Don Birnam), Jane Wyman (Helen St. James), Phillip Terry (Wick Birnam) et Howard Da Silva (Nat). Paramount Pictures, 1945. DVD. Universal Pictures France, 2007.

Topaz. Réal. Alfred Hitchcock. Scénario de Samuel Taylor. Avec Frederick Stafford (André Devereaux), Dany Robin (Nicole Devereaux), John Vernon (Rico Parra) et Karin Dor (Juanita de Cordoba). Universal Pictures, 1969. DVD. Universal Pictures France, 2001.

Torn Curtain. Réal. Alfred Hitchcock. Scénario de Brian Moore. Avec Paul Newman (Professor Michael Armstrong), Julie Andrews (Sarah Sherman), Hansjörg Felmy (Heinrich Gerhard) et Wolfgang Kieling (Hermann Gromek). Universal Pictures, 1966. DVD. Universal Pictures France, 2001.

\section{NOTES}

1. Contrairement à Wilder, Hitchcock n'est pas scénariste de son film, et c'est Samuel A. Taylor qui en a signé le script (on notera que Taylor a également co-écrit celui de Sabrina [1954] pour Wilder, avec Wilder lui-même et Ernest Lehman). Toutefois, on sait qu'à l'époque de Topaz et 
depuis des années déjà, Hitchcock possédait un total contrôle sur l'écriture de ses longs métrages et que les auteurs de ses scripts travaillaient en étroite collaboration avec lui et avec son épouse scénariste, Alma Reville, sans que cette dernière soit nécessairement créditée au générique.

2. "It was like making a picture in Pompeii with all the lava coming down. Khrushchev was even faster than me and Diamond. We had to make continual revisions to keep up with the headlines".

3. « Film makers are vulnerable to this kind of risk. A situation, a political mood changes in the course of your making a film and things are not the same by the time you finish the picture as they were when you started. If you write a newspaper piece, it appears the next day. [...] But film makers who do a contemporary story have to pray that the situation that they are dealing with in the projected movie will still be valid a year and a half in the future. Otherwise people may say that you are guilty of bad taste [...]».

4. On notera que dans une précédente réalisation d'Hitchcock, Notorious (Les Enchaînés, 1946), la tasse de café empoisonné est visuellement démesurée par rapport au personnage vulnérable d'Alicia (Ingrid Bergman), et que des bouteilles en amorce viennent régulièrement "écraser » l'écrivain de The Lost Weekend (Le Poison, Billy Wilder, 1945), comme pour (lui) rappeler l'emprise que l'alcool a sur lui.

5. «[...] any world that can produce the Taj Mahal, William Shakespeare and striped toothpaste can't be all bad.»

6. «Instead of dollars, you would accept three-week tour of Bolshoi Ballet? " - «Please. No culture, just cash.»

7. « Ninotchka's transformation in accepting the hat is not so much an acceptance of capitalist ways - she never loses her political convictions as the Ninotchka of Silk Stockings does - but rather the acceptance of her own foolishness ».

8. « Won't school soon open? » - «In Georgia? You never know.»

9. « erasing the distinction between moral and immoral ».

\section{RÉSUMÉS}

One, Two, Three de Billy Wilder (Un, deux, trois, 1961) et Topaz d'Alfred Hitchcock (L'Étau, 1969) sont deux films qui n'ont pas rencontré un vif succès à leur sortie et que les critiques n'ont jamais encensés. Pourtant, ils ne sont pas aussi mineurs qu'on pourrait le croire: leur intrigue loufoque pour le premier, plutôt nébuleuse pour le second-cache des prises de position idéologiques subtiles et inattendues de la part de réalisateurs d'ordinaire discrets quant à leurs opinions politiques. Cet article s'attache à étudier les moyens mis en œuvre par Wilder et Hitchcock, tant au niveau du scénario que de la mise en scène, pour illustrer leur vision du capitalisme et du communisme. Certes, les deux cinéastes critiquent l'héritage révolutionnaire ; mais ils ont également l'audace, en pleine guerre froide, de se montrer peu indulgents à l'égard du modèle proposé par leur pays d'adoption.

Billy Wilder's One, Two, Three (1961) and Alfred Hitchcock's Topaz (1969) were not successful when released. This essay argues that in spite of this lack of critical acclaim, they are worth revisiting today: their respective plots-the former, farcical, the latter, rather nebulous-dissimulate subtle ideological stances all the more unexpected because neither Wilder nor Hitchcock were known for making their political views public. The aim of this essay is to study the devices used by both 
whether in their script or filming, to illustrate their vision of capitalism and communism. The two directors did criticize the revolutionary heritage-but in the midst of the Cold War, they also took an unsupportive attitude towards the proposed model of their adopted country.

INDEX

Keywords : Alfred Hitchcock, Billy Wilder, Topaz, One, Two, Three, Berlin Wall, Cuba, Cold War, revolution

Thèmes : Hors-thème

Mots-clés : Alfred Hitchcock, Billy Wilder, L'Étau, Un, deux, trois, Mur de Berlin, Cuba, guerre froide, révolution

\section{AUTEUR}

JULIE MICHOT

Université du Littoral-Côte d'Opale 